Meta

Journal des tradlucteurs

Translators' Journal

\title{
Traduction littérale ou traduction libre ?
}

\section{Jean Darbelnet}

Volume 15, numéro 2, juin 1970

URI : https://id.erudit.org/iderudit/002478ar

DOI : https://doi.org/10.7202/002478ar

Aller au sommaire du numéro

Éditeur(s)

Les Presses de l'Université de Montréal

ISSN

0026-0452 (imprimé)

1492-1421 (numérique)

Découvrir la revue

Citer cet article

Darbelnet, J. (1970). Traduction littérale ou traduction libre ? Meta, 15(2),

88-94. https://doi.org/10.7202/002478ar d'utilisation que vous pouvez consulter en ligne.

https://apropos.erudit.org/fr/usagers/politique-dutilisation/ 


\section{trraduction lirtuérale}

\section{() U}

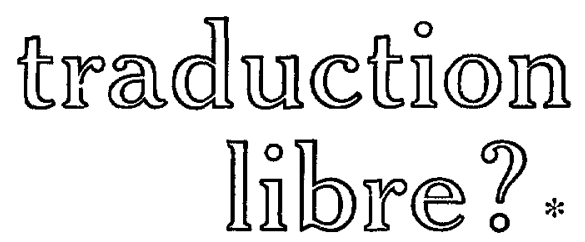

Il y a quelque temps, nous avons exposé ici même (META, XIV, 3 (1969) : 135-140) une certaine conception de la traduction raisonnée et nous avons terminé en citant Hilaire Belloc, pour qui le traducteur ne doit être asservi ni à la forme ni à la longueur. C'est là un point de vue auquel nous sommes fort attaché, mais qui n'a pas toujours prévalu, les belles infidèles relevant d'une autre conception, et qui est encore loin d'être celui de tous les traducteurs, en particulier au Canada.

Le présent article s'efforce d'exposer pourquoi la conception que Belloc se fait de la traduction est la bonne, et pourquoi la question : «Faut-il traduire littéralement ou librement? » est une question mal posée, la réponse étant : ni littéralement ni librement, car l'essentiel est de traduire exactement, et il importe peu que ce soit en gardant la forme ou en la modifiant.

Il se trouve qu'à environ un siècle d'intervalle deux grands écrivains se sont prononcés sur ce point de telle sorte que leurs vues opposées permettent de délimiter le champ du débat. Dans la préface de sa traduction d'Hamlet, Gide condamne la « littéralité » des traductions «consciencieuses et exactes» au point d'en être incompréhensibles. Il recommande une certaine tricherie comme antidote et il pose en principe que le bon traducteur doit être un mâ̂tre écrivain, ce qui paraît difficile à une époque où le recrutement massif des traducteurs est devenu une nécessité. On trouvera dans la Stylistique comparée du français et de l'anglais, p. 267-269, une réfutation des idées de Gide en matière de traduction.

Celles de Chateaubriand se situent à l'opposé, si l'on en juge par ce qu'il a écrit dans la préface de sa traduction du Paradis perdu : «La traduction littérale me paraît toujours la meilleure : une traduction interlinéaire serait la perfection du genre, si on lui pouvait ôter ce qu'elle a de sauvage. "On peut dire que le point de vue de Belloc se situe entre ces deux extrêmes. En un sens, Belloc paraît plus près de Gide. Il est, lui aussi, contre la littéralité, mais rien dans ce qu'il préconise ne fait allusion à la tricherie proposée par Gide. L'opinion

\footnotetext{
* D'après une conférence prononcée à l'Université d'Ottawa le 22 octobre 1969.
} 
de Chateaubriand est aux antipodes de la sienne, encore que l'«interlinéarité » n'interdise pas nécessairement les changements de forme. Mais il est bien évident qu'une traduction interlinéaire ne peut guère être plus longue que l'original sous peine de perdre son utilité. Or, longueur et forme se tiennent, car qui refuse de faire plus long - ou plus court — quand le sens l'exige, ne pourra guère s'éloigner du mot à mot.

Appliquée à la traduction, la stylistique comparée, telle qu'elle est présentée dans l'ouvrage mentionné plus haut, n'aurait guère de raison d'exister ou, en tout cas, aurait beaucoup moins d'ampleur, s'il suffisait de traduire littéralement pour bien traduire, comme semble le croire Chateaubriand. La traduction littérale n'est, en effet, qu'un des sept passages proposés pour passer d'une langue à l'autre, et si la transposition et la modulation s'opèrent à l'intérieur de segments qui sont le plus souvent à peu près de la même longueur, par contre, l'équivalence et l'adaptation entraînent souvent des changements de longueur aussi bien que de forme. Mais les préceptes que propose la stylistique comparée vont encore à l'encontre d'habitudes invétérées. Aussi ne suffit-il pas d'observer que ceux qui n'osent pas ne pas traduire littéralement auraient l'impression, s'ils s'y risquaient, de s'écarter du sens, il faut montrer que cette crainte est injustifiée et que loin d'offrir la sécurité, la traduction littérale expose à des contresens, sans compter les déformations qu'elle fait subir à la langue d'arrivée.

Si l'on refuse le critère de la littéralité, critère qui découle de la croyance que la littéralité garantit l'exactitude, il faut pouvoir s'appuyer sur autre chose, et la recherche d'autres critères amène à réfléchir aux exigences d'une bonne traduction. Essentiellement une traduction doit, pour être à l'abri de tout reproche : 1) transmettre exactement le message de l'original; 2) observer les normes grammaticales de son temps ; 3) être idiomatique ; 4) être dans le même ton que l'original (équivalence stylistique) ; 5) être pleinement intelligible pour le lecteur qui appartient à une autre culture (adaptation culturelle).

On remarquera que dans ce qui précède, la structure n'intervient pas, et à la réflexion, on se rend compte qu'il est normal qu'il en soit ainsi. Les structures ou agencements de mots (et les mots sont à leur tour des structures) véhiculent une pensée. Le traducteur doit se rendre compte et ne jamais perdre de vue qu'il n'a pas le droit de sacrifier la pensée à la structure et que, en revanche, il ne doit jamais hésiter à sacrifier la structure pour sauvegarder la pensée. Nous établissons ainsi une dichotomie structure/pensée que le traducteur est tenu d'avoir toujours présente à l'esprit. Elle n'est pas une idée préconçue, elle est dans la nature des faits. Tout énoncé se compose de mots choisis et disposés pour dire quelque chose, tout énoncé est de la pensée structurée à l'aide de mots et de leur agencement. Changer la structure de l'énoncé à l'intérieur de la même langue risque, certes, d'en modifier l'effet et même le sens, mais le problème n'est pas le même quand on traduit, car les langues diffèrent suffisamment pour imposer des structures différentes à l'expression de la même idée. C'est ce que, consciemment ou inconsciemment, refusent d'admettre les tenants de la traduction littérale, 
qui sont au fond convaincus qu'en «collant» au texte, ils ne peuvent pas se tromper. Ce n'est pas au texte en tant que forme matérielle qu'il faut «coller», mais au sens qui se dégage du choix et de la disposition des mots. Toutes les fois que l'on traduit littéralement sans comprendre, on s'expose, contrairement à une croyance encore trop répandue, à trahir le sens du message.

Étant donné la persistance des traductions littérales, il n'est sans doute pas superflu de s'arrêter à des exemples qui fournissent la preuve irréfutable que la traduction littérale, souvent peu idiomatique, constitue parfois un contresens. En d'autres termes, il est possible de trahir la pensée en calquant la structure. Nous donnons ci-dessous la phrase anglaise, sa traduction littérale entre parenthèses et sa traduction exacte. On remarquera que la littéralité aboutit, dans les exemples considérés, à une forme parfaitement correcte et même idiomatique.

He has been in Paris for two years.

(Il a été à Paris pendant deux ans.)

Il est à Paris depuis deux ans.

He is sure to come.

(Il est sûr de venir.)

Il viendra sûrement.

Be sure he understands what he has to do.

(Soyez sûr qu'il comprend ce qu'il a à faire.)

Assurez-vous qu'il comprend ce qu'il a à faire.

Point n'est besoin de signaler que les énoncés entre parenthèses correspondent respectivement aux phrases suivantes. He was in Paris for two years. He is sure he can come. You may be sure that he understands what he has to do.

Les exemples de traduction littérale que l'on vient de voir relèvent de la catégorie des faux amis de structure qui comprennent non seulement des constructions syntaxiques mais aussi des unités de vocabulaire. Aucun traducteur expérimenté ne confondra pomme de pin et pineapple malgré le parallélisme des éléments de même sens, ou encore lodger (celui qui est logé) et logeur (celui qui loge), mais simple soldier a été traduit par simple soldat, et il n'est pas sûr que l'on aperçoive toujours la nuance qui sépare distaste de dégoût. Récemment l'auteur de cet article s'est vu dans la nécessité de signaler aux membres d'une société savante que sciences naturelles ne désigne pas la même catégorie de sciences que natural sciences. Sur un plan légèrement différent on constate que, dans la langue épistolaire, Dear Friend et Cher ami ne correspondent pas à des situations identiques.

Il se peut que dans les cas ci-dessus la paresse, l'inattention soient autant à l'origine de ce genre de méprise que la peur de s'écarter du mot à mot. Quoi qu'il en soit, le danger de la littéralité existe et il importe que les futurs traducteurs en soient avertis. Un moyen simple de leur faire toucher du doigt les différences de structure qui rendent compte d'un même message est de leur proposer une phrase dont la traduction idiomatique entraîne le chamboulement des éléments, et de leur faire retrouver dans la traduction les unités reconnues dans l'original. C'est, comme on le sait, la technique du découpage. Dans la phrase suivante, les unités sont numérotées et séparées par des barres verticales :

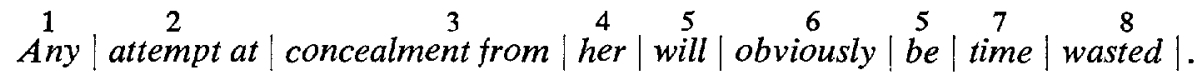


Les mots will et be forment une unité parce qu'ils représentent le verbe to be au futur. La question se pose d'ailleurs de savoir si ce futur passe en français. En fait, c'est un présent d'habitude, mais l'essentiel n'est pas là. En traduisant, on bute contre le fait qu'à concealment ne correspond pas de substantif commode en français et que ce mot se traduit beaucoup mieux par un verbe. Cela oblige à d'autres transpositions dont le résultat est celui-ci :

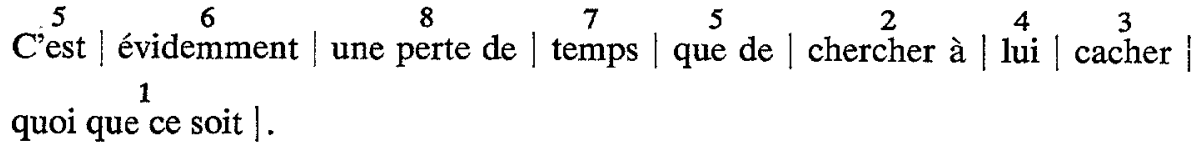

On aboutit ainsi à un bouleversement complet des unités de l'original mais aucune n'est perdue et il semble même, à première vue, qu'il y en ait une de trop. Le est de c'est correspond bien à will be, mais c'est... que est une mise en relief dont l'équivalent est l'accent de phrase qui tombe sur any.

Les exemples qui précèdent ont montré que la traduction littérale n'est pas nécessairement exacte et que, de plus, il lui arrive de couler la matière de l'énoncé dans un moule qui n'est pas fait pour lui, comme c'eût été le cas si nous avions essayé de rendre mot à mot la phrase commençant par Any attempt at...

Il convient aussi de noter en passant les cas où c'est la situation qui décide du choix des mots et où l'on emploie automatiquement une formule. Il faut alors se garder d'introduire dans la langue d'arrivée une phrase qui n'a pas cours dans la situation que décrit l'original. Il est bon, avant de se laisser aller à traduire servilement, de se demander ce qu'on dit dans l'autre langue en pareil cas. Dans un magasin tenu par des anglophones, May I help you? est la façon rituelle de s'adresser à un client. Rien ne s'opposerait que l'équivalent français soit : Puis-je vous aider? Rien sauf l'usage qui impose la formule Est-ce qu'on s'occupe de vous? ou Vous désirez? Nous avons ici le cas d'une phrase parfaitement correcte et qui se dit souvent, mais pas dans la situation du vendeur s'adressant au client.

Ainsi donc, la traduction littérale est trop rigide pour tenir compte de ce qui se cache derrière les mots. Mais son principal défaut est d'aller souvent à l'encontre de ce qu'on appelle parfois le génie de la langue. Nous avons déjà vu dans un article précédent ce qu'il faut entendre par ce terme et il n'est pas nécessaire de rappeler qu'une phrase peut être grammaticale sans être pour cela idiomatique. $\mathrm{Ni}$ en français ni en anglais on ne dira : « un livre est sur la table», «a book is on the table », et en français, Il reste beaucoup à faire est la traduction idiomatique de Much remains to be done, beaucoup, en tant que nominal sujet, exprimant la pluralité plutôt que l'abondance d'une matière. Mais il y a plus. Il y a la préférence secrète d'une langue pour tel ou tel mode d'expression. Dans une phrase comme « les couleurs disparaissent $»$, la traduction de disparaître par discippear paraîtra insolite à un anglophone, qui attend plutôt un verbe concret comme to fade. Le vent entre ou arrive en français, mais non en anglais où l'on marque la marche du vent au moyen de to blow. Certains, obsédés par le démon 
de la littéralité, hésiteront à remplacer l'abstrait par le concret ou vice versa, oubliant ainsi que, pas plus que les individus, les langues ne doivent forcer leur talent, sauf quand elles sont maniées par un grand écrivain. D'ailleurs nous avons tendance à juger les mots d'une autre langue autrement que les personnes qui la parlent depuis leur naissance. Nous leur prêtons des harmoniques qu'ils n'ont pas forcément. Pour un anglophone, ou pour un francophone qui s'est anglicisé, le verbe entrer paraît bien faible, bien incolore, et en effet c'est un MOT SIGNE et non un MOT IMAGE, mais on admettra qu'il est mis en valeur dans la phrase suivante par laquelle le Monde a titré la nouvelle d'un accident survenu, il y a plusieurs années, aux Vingt-quatre heures du Mans : "La voiture de von Trips entre dans la foule ». L'effet d'horreur est aussi accusé qu'avec le verbe anglais to crash.

Un autre défaut de la traduction littérale est de forcer les mots qui se correspondent en gros d'une langue à l'autre à avoir toujours le même comportement. Or ce comportement n'est, le plus souvent, que partiel. En particulier, l'équivalence des sens propres de deux homologues n'entraîne pas nécessairement celle de leurs sens figurés. Sin veut dire «péché », mais, employé au figuré, il se rend plutôt par faute impardonnable. Pocket s'emploie au figuré en anglais dans une acception que poche semble refuser, et l'on traduira unconquered pockets of ignorance and prejudice par des îlots non résorbés d'ignorance et de préjugés. Tragedy au sens figuré est un drame, un accident, un événement tragique, mais non une tragédie, car ce mot en français sert surtout à désigner un genre littéraire.

À cet égard, le langage des métaphores est un domaine que la linguistique différentielle n'a exploré qu'en partie. On peut considérer en simplifiant un peu les choses, qu'on y trouve trois types d'équivalences : 1) les métaphores identiques ; 2) les métaphores qui n'utilisent pas les mêmes termes de comparaison; 3) les métaphores qui équivalent à des vues directes, ou vice versa, ce qui veut dire que l'une des deux langues est dépourvue d'une métaphore pour rendre l'idée dont il s'agit. Voici un exemple de chaque catégorie :

1) to wash one's hands of it = s'en laver les mains.

2) it went like clockwork = cela a marché comme sur des roulettes.

3) to spread = faire tache d'huile.

Le Harrap ne cherche pas à rendre faire tache d'huile par un équivalent métaphorique, et il a raison. Au Canada on dit, du lait qu'on laisse trop longtemps sur le feu, qu'il «renverse». En France, il se sauve et dans les pays de langue anglaise, it boils over. Nous avons là, respectivement, des exemples de VUE DIRECTE (renverser), de MÉTAPHORE (se sauver) et d'IMAGE DIRECTE (to boil over). Cette terminologie a de quoi surprendre, car on tend à employer indifféremment métaphore et image dans les études littéraires. Nous avons intérêt en stylistique comparée à garder à métaphore son sens habituel et à réserver image directe, comme l'a proposé $M$. Malblanc, pour les notations qui sont en prise directe sur le réel. Dans to boil over, il n'y a pas de métaphore, il y a seulement le film de l'action, et l'on voit le lait bouillir et passer finalement par-dessus le bord de la casserole. Cette distinction est une de celles qui permettent de caractériser les écarts inévitables entre l'original et une bonne traduction, dès l'instant qu'on n'est pas l'esclave de la littéralité. La différence essentielle entre la métaphore et 
l'image directe est que celle-ci ne comporte pas de comparaison et rend directement le réel. Dans l'Étranger de Camus, Meursault entend de sa prison les bruits de la ville et en particulier la plainte des tramways dans les hauts tournants de la ville. Cette notation a été rendue en anglais par the screech of street-cars at the steep corners of the upper town. Instinctivement, M. Stuart Gilbert a senti que plainte, au sens figuré, demandait à être traduit par un des nombreux mots concrets dont l'anglais dispose pour distinguer les bruits, et il a remplacé une métaphore par une image directe, sans vraisemblablement avoir conscience de cette distinction.

Toute langue utilisée par une ethnie est liée à une culture, au sens que les sociologues donnent à ce mot, et le respect des faits de culture s'accommode mal de la littéralité. Une simple phrase telle que He walked two blocks east sera, traduite littéralement, difficilement compréhensible pour un Français qui ne connaît pas l'Amérique. Il est vrai qu'elle sera immédiatement comprise d'un Canadien français, justement parce que les Canadiens qu'ils soient francophones ou anglophones vivent en grande partie à l'américaine. Si nous considérons maintenant l'intégrité de la langue et non la clarté du message, le problème est à peu près le même pour les Québécois et les Français, si l'on veut, comme il se doit, éviter «marcher deux blocs». Il s'agit en fait d'une distance que l'on peut évaluer en mesure itinéraire d'après le contexte. On pourra dire vers l'est. Mais il est à remarquer que ni les Anglais ni les Français ne s'orientent d'après les points carclinaux à l'intérieur d'une ville, de sorte que, pour des lecteurs européens, il vaut mieux prendre un point de repère pour marquer l'orientation. Ainsi, l'avis que l'on voit à la gare Windsor pour indiquer qu'il y a un restaurant « at the south end of the concourse », représente un fait de culture nord-américain. La traduction littéraire est satisfaisante pour un Canadien ; elle paraîtra insolite à un Européen, pour qui il serait plus naturel d'indiquer la sortie d'après la rue sur laquelle elle donne. Notons aussi qu'il y a des choses qui ne se traduisent pas et qu'il ne faut pas essayer de traduire. Bon appétit! est un souhait français qui ne passe pas en anglais.

Dans les cas que nous venons de voir il y a adaptation de la traduction à la culture du lecteur étranger. Cette adaptation comporte parfois des omissions et des ajouts. Cela n'intéresse pas le lecteur français que les wagons de marchandises dans l'un desquels le héros de $A$ Farewell to Arms s'apprête à sauter s'appelle en Amérique gondola. Par contre l'expression version autorisée, parfaitement claire pour un anglophone, a besoin d'être explicitée pour un Français. Il faut ajouter : de la Bible, à moins que ce qui précède immédiatement indique bien de quoi il s'agit. Ni l'omission de gondola, ni l'adjonction d'un complément de nom à version autorisée ne prête à discussion ; sur le plan pratique l'une et l'autre se justifient facilement. Mais il y a des adaptations facultatives que l'on accepte ou que l'on écarte suivant l'école à laquelle on appartient. Dans quelle mesure le lecteur tient-il a être dépaysé, à sentir qu'il n'est pas chez lui ? Préfère-t-il au contraire que l'adaptation soit complète pour que rien ne lui rappelle l'identité «culturelle » 
de l'original ? Faut-il, pour prendre un exemple banal, convertir en milles les kilomètres dont il est question dans un roman français ? Chacun décide suivant sa doctrine en matière d'adaptation, mais la tendance de l'époque est de mettre de la couleur locale partout où il se peut. C'est ainsi que pendant la guerre des journalistes français disaient la R.A.F. plutôt que l'aviation anglaise. Certains sont même allés jusqu'à appeler Aachen la ville d'Aix-la-Chapelle, mais c'est là un autre sujet qui mériterait d'être traité à part.

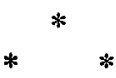

Une série d'exemples nous ont permis de montrer les déficiences de la traduction littérale. On peut, en guise de conclusion, reprendre ces «manques » qu'une bonne traduction ne doit pas laisser subsister et en faire un tableau auquel il suffirait de se reporter pour juger de la qualité des traductions et en quelque sorte la mesurer.

Il est peu probable qu'une traduction n'ait aucun sens. Par contre, il peut arriver, comme nous l'avons vu, qu'elle renferme des contresens. Ce serait le premier et le plus grave manquement, à partir duquel on peut situer les autres. Cela donne, en prenant la phrase comme unité d'examen :

La traduction (de telle phrase) 1) ne garde pas le sens de l'original ;2) fait violence à la structure de la langue d'arrivée ; 3) est possible structuralement, mais n'est pas idiomatique ; 4) ne garde pas le ton de l'original ; 5) renferme une image que n'utilise pas la langue d'arrivée ; 6) fait allusion à un fait de culture que le lecteur risque de ne pas comprendre.

Il est facile de trouver dans ce qui précède les exemples correspondant à chacune des déficiences énumérées ci-dessus. L'utilité de cette codification est de fixer les limites précises à la liberté qu'on semble se donner en n'étant pas littéral ; mais cette liberté n'est pas arbitraire, elle est en fait l'obligation où l'on est de respecter des différences dûment reconnues entre les deux langues. C'est pourquoi, reprenant la question du début, on peut répondre qu'il ne s'agit jamais de traduire littéralement ou librement, que la seule chose qui compte est de traduire exactement, c'est-à-dire en respectant d'une part le sens et la tonalité de l'original (articles 1 et 4 ), et d'autre part l'intégrité de la langue d'arrivée sous le rapport de la structure, du génie, des images et des faits de culture (articles 2, 3, 5 et 6).

JeAN Darbelnet 